\title{
CHANGES IN ELECTROMYOGRAPHIC PARAMETERS DURING CYCLING EXERCISE AT CONSTANT INTENSITY
}

\author{
L. Lepasalu, J. Ereline, H. Gapeyeva, T. Kums, M. Pääsuke \\ Institute of Exercise Biology and Physiotherapy, \\ Estonian Centre of Behavioral and Health Sciences, \\ University of Tartu, Estonia
}

\begin{abstract}
The aim of this study was to evaluate the changes in surface electromyography (sEMG) parameters during 30-minute cycling exercise $(250 \mathrm{~W})$ at constant intensity in laboratory conditions. Ten male cyclists with the mean $( \pm \mathrm{SE})$ age of $23.8 \pm 3.7$ years participated. The sEMG power spectrum median frequency (MF) of rectus femoris, vastus lateralis, biceps femoris and erector spinae muscles, heart rate and the subjective rate of fatigue by Borg scale were measured in the beginning, during and at the end of exercise. Maximal voluntary contraction (MVC) force of the knee extensor muscles was assessed before and after the end of exercise. A significant decrease $(p<0.05)$ in sEMG power spectrum MF for erector spinae muscle at the end of exercise compared to the initial level was found, whereas the knee extensor and flexor muscles revealed no significant changes in this parameter. The subjective rate of fatigue increased moderately during the cycling exercise $(p<0.05)$. The heart rate was stable during the exercise, remaining predominantly in the aerobic zone. After the exercise, MVC force was significantly $(p<0.05)$ decreased compared to the pre-exercise level.

We concluded that based on sEMG power spectrum MF, 30minute cycling exercise at constant intensity caused a marked fatigue of erector spinae muscle with no significant fatigue-induced changes in knee extensor and flexor muscles. A marked decrease of voluntary force capacity of knee extensor muscles was found after exercise. A
\end{abstract}


moderate subjectively assessed fatigue was established at the end of exercise.

Key words: neuromuscular fatigue, cycling exercise

\section{INTRODUCTION}

Muscle fatigue can be defined as the failure to maintain a required or expected power output $[3,9]$. This impairment can include peripheral (e.g. neuromuscular propagation, excitation-contraction coupling, myofibrillar activity) and central processes [3, 9, 18]. Numerous studies in this field have focused on measuring neuromuscular fatigue in exercises lasting from 30 minutes to several hours in the last decade $[8,14]$. The aetiology of muscle fatigue is very complicated, especially in endurance sports $[10,14]$. Many authors describe fatigue, depending on work intensity and the nature of fatigue. There is a firm standpoint that fatigue is the body's defensive reaction that regulates the mobilization of reserves $[9,16,17,18]$. It is believed that the muscle contraction type (isometric, concentric, eccentric) and the training type (endurance, strength, power) of the neuromuscular system cause neuromuscular functional decrease in performance. However, there is little information how their relationship affects the neuromuscular fatigue development. Surface electromyography (sEMG) analyzes the activity of skeletal muscles by amplitude and frequency parameters. Muscle fatigue has been often evaluated by assessment of median frequency (MF) [1].

Several neuromuscular fatigue studies have dealt with mechanical production of muscular force in sprinting exercises on cycle ergometers $[6,8]$ and there have also been investigations on training quality relationship with muscle strength and sEMG activity [16]. It has been found that the development of neuromuscular fatigue after prolonged exercise (30 minutes to several hours) is different in cyclists, runners or skiers [14]. This is explained by the adaptation of the neuromuscular system to the muscle contraction that is mainly used in daily sports activities. However, our knowledge of the effect on fatiguing submaximal dynamic exercise to organism functions among athletes of different sports is insufficient. Thus, there is a need for further studies at athletes representing different sports before and after fatiguing exercise. 
The purpose of this study was to evaluate the development of fatigue during 30-minute cycling exercise in laboratory conditions at constant intensity. More specifically, we registered the sEMG power spectral MF of rectus femoris, vastus lateralis, biceps femoris and erector spinae muscles, as well as heart rate and the subjective rate of fatigue on Borg scale of the participants during the exercise. Also, fatigue-induced changes in maximal voluntary isometric contraction (MVC) force of the knee extensor muscles were measured.

\section{METHODS}

\section{Subjects}

Ten male cyclists participated in this study. The mean $( \pm \mathrm{SE})$ age, body height, body mass and body mass index of the subjects were $23.8 \pm 1.2$ years, $183.5 \pm 2.2 \mathrm{~cm}, 74.5 \pm 2.1 \mathrm{~kg}$ and $22.1 \pm 0.5 \mathrm{~kg} / \mathrm{m}^{2}$, respectively. The cyclists were the best road cyclists in Estonia. Two cyclists belonged to the Estonian professional cycling team, three cyclists belonged to a French amateur team and five participants were freelancers. The subjects had regularly trained cycling for $11.2 \pm 1.3$ years prior to the study. The mean training volume among subjects was $16.7 \pm 0.8$ training hours per week and they had $6.2 \pm 0.1$ training days per week. Their written informed consent was obtained. The study was carried out at the Laboratory of Kinesiology and Biomechanics of the University of Tartu in June 2010.

\section{Experimental design}

The subjects performed 30-minute cycling test using their own racing bicycles, shoes and pedals. Bicycle was fixed to the ergometer Taxc Cosmos. Each subject carried out a standardized warm-up period before each testing session, consisting of 5-minute cycling at constant $100 \mathrm{~W}$ intensity. Each subject performed one 30-minute cycling trial at cadence 90 per minute at constant power output $(250 \mathrm{~W})$. The heart rate during the test was registered by Polar heart rate tester (Finland). The sEMG electrodes were placed to subject's dominant body side on four muscles: rectus femoris, biceps femoris, vastus lateralis, erector spinae. In order to prevent shifting, wires between the electrodes and the computer were secured to the skin with adhesive tape. 
sEMG activity was recorded with surface electrodes T-601. Low impedance between the electrodes was obtained by light abrasion of the skin. Electrodes were coated with electrolytic gel and fixed lengthwise over the middle of the muscular belly. sEMG power spectrum MF of the measured muscles was recorded by electromyograph ME6000 (Kuspio, Finland) for $60 \mathrm{~s}$ periods for 3 times: in the beginning $(0-1 \mathrm{~min})$, in the middle $(14-15 \mathrm{~min})$ and at the end (29$30 \mathrm{~min}$ ) of exercise.

The subjective rate of fatigue on Borg scale of the participants was registered before the test and for two times during the 30-minute cycling test: at $15 \mathrm{~min}$ and at $30 \mathrm{~min}$. Fatigue non-existed at number 6 and fatigue was maximal at number 20 [4]. Every odd number was marked by words. The scale deficiency is its subjectivity and dependence on the subject's self-realization level, therefore it is suitable for experienced athletes.

MVC force of the knee extensor muscles was measured using a custom-made dynamometric chair. During the test, the subjects were instructed to react to the light signal (ignition of the signal lamp, placed $1.5 \mathrm{~m}$ from the subject) as quickly and as strongly as possible by extending the right leg against a cuff fixed to a strain gauge system, to maintain the maximal effort as long as the signal was on $(2 \mathrm{~s})$ and to relax the muscles after the disappearance of the signal. The subjects were motivated by visual feedback and verbal encouragement. Three maximal attempts were recorded and the best result of MVC force was taken for further analysis. The MVC force was measured before and immediately after 30-minute cycling exercise.

\section{Statistical analysis}

Data are expressed by means and standard error of mean $( \pm \mathrm{SE})$. The significance of the changes for the group of subjects in the beginning, in the middle and at the end of exercise was analyzed by the student $t$ test. A level of $p<0.05$ was selected to indicate statistical significance.

\section{RESULTS}

sEMG power spectrum MF was significantly decreased $(p<0.05)$ at the end of exercise compared to initial level only for erector spinae muscle. For other measured muscles, the changes in MF were not significant ( $p>0.05$; Figure 1). The subjective rate of fatigue increased 
during cycling exercise, whereas this parameter was higher $(p<0.05)$ in the middle and at the end of exercise compared to the beginning of the test (Figure 2). The heart rate was stable during the exercise, remaining predominantly in the aerobic zone (Figure 3). MVC force of the knee extensors in cyclists was significantly decreased $(p<0.001)$ after the end of 30-minute cycling test at constant intensity compared to the pre-exercise level (Figure 4).

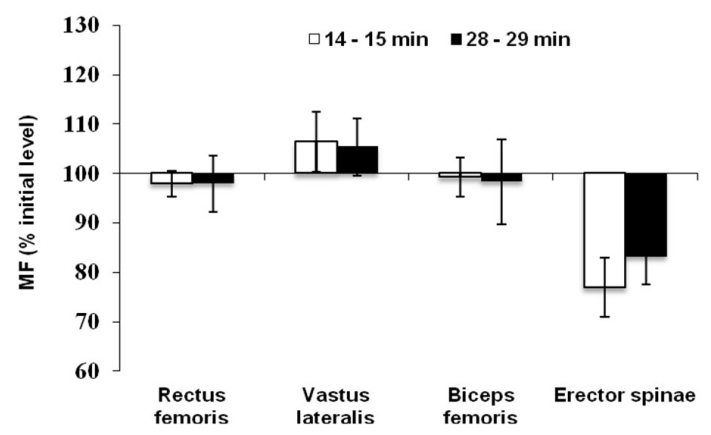

Figure 1. Median frequency (MF) of the electromyography power spectrum recorded from rectus femoris, vastus lateralis, biceps femoris and erector spinae muscles during 30-minute cycling exercise (mean $\pm \mathrm{SE}$ ). Values are presented in percent from the initial level in the beginning of the test, taken as $100 \%$. $* *$ Significantly $(\mathrm{p}<0.01)$ different from the initial level.

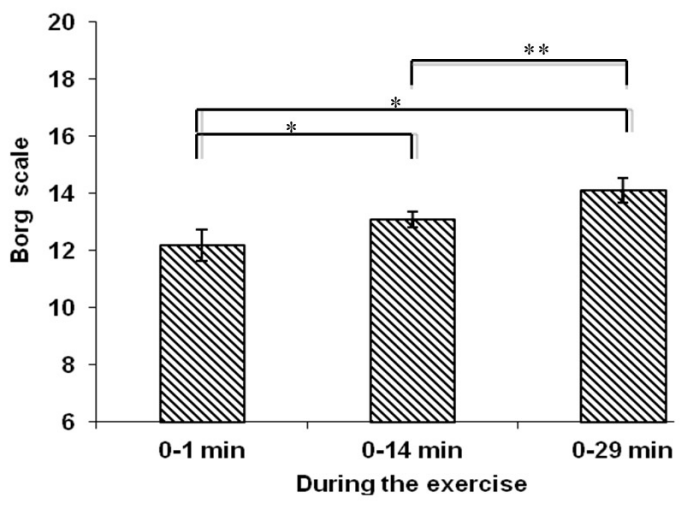

Figure 2. Subjective rate of fatigue evaluated by BORG scale during 30minute cycling exercise (mean $\pm \mathrm{SE}$ ). ${ }^{*} \mathrm{p}<0.05 ;{ }^{*} \mathrm{p}<0.01$. 


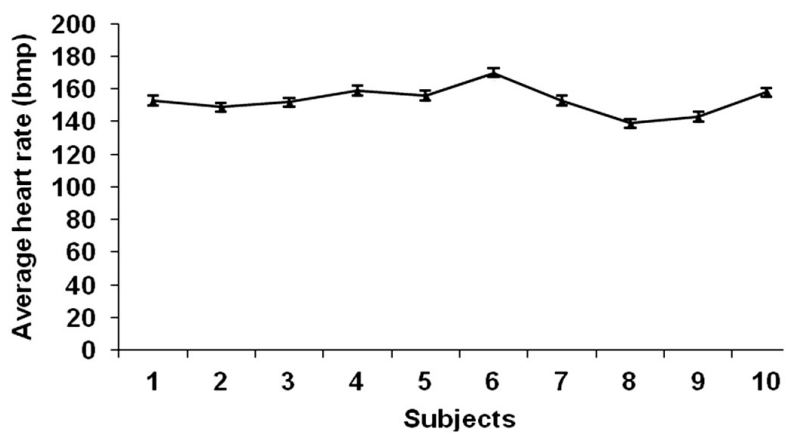

Figure 3. Average heart rate (HRT) of subjects during cycling test (mean $\pm \mathrm{SE})$.

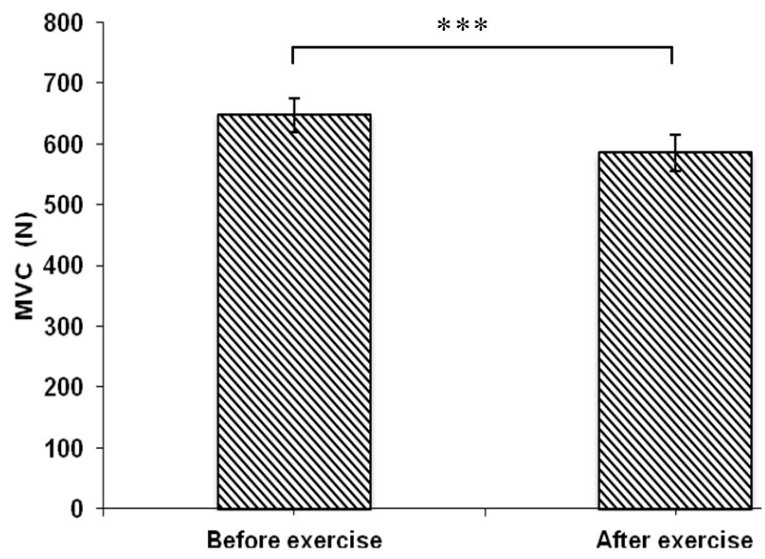

Figure 4. Isometric maximal voluntary contraction (MVC) force of the knee extensor muscles before and after exercise (mean $\pm \mathrm{SE}$ ). ${ }^{* * *} \mathrm{p}<0.001$.

\section{DISCUSSION}

The main results of this study were that 30 -minute cycling exercise at constant intensity caused: (a) a significant fatigue of erector spinae muscle during cycling exercise evaluated by decrease of sEMG power spectrum MF and (b) a marked reduction of maximal isometric voluntary force generation capacity of the knee extensor muscles immediately after the end of exercise. 
sEMG analysis during the cycling exercise showed that MF of erector spinae muscle was decreased by $16.9 \%$ at the end of exercise compared to the beginning of exercise, whereas MF decrease for other measured knee extensor and flexor muscles was smaller (from 2 to $5 \%$ ). In general, it has been reported that sEMG power spectrum MF decreases as muscle fatigue increases. During sustained fatiguing submaximal contraction, decrease in s EMG power spectrum MF with advancing contraction time has been demonstrated in numerous previous studies $[7,12]$. This temporal pattern in the sEMG signal was also observed in the present study. The factors that may contribute to decrease of MF (spectral compression) are decreased muscle fibre conduction velocity [19] and synchronization of the motor units firing [2]. A tendency towards synchronization of motor units reflects a common presynaptic input to motoneuron pool. In the opinion of many authors, motoneuron shifts are caused by a decrease in the membrane conduction velocity occurring during the fatiguing process due to local metabolic changes and ion shifts in the muscles [5].

In this study, the subjective rate of fatigue assessed by Borg scale increased from 12 in the beginning to 14 points at the end of cycling test $(16.7 \%)$. The cyclists subjectively evaluated this cycling test as an easy exercise. During the test, with increasing load, the rate of subjective fatigue $(6.6 \%)$ increased to the hard zone. This meant that cyclists felt fatigue, but were able to continue with the given load. At the end of exercise cyclists still evaluated the subjective rate as "hard", being by $14.8 \%$ higher compared to the pre-fatigue level. The Rating Scale of Perceived Exertion from 6 to 20 [4] is the most frequently used identification technique of perceived exertion. PérezLandaluce et al. [15] found that the Borg scale is a practical measure to prescribe exercise-training intensities in professional, amateur and young cyclists. Fatigue during endurance exercise at constant intensity depends on the conformity of heart rate at given load nature and duration.

In the present study the subjects' heart rate was monitored to assure physiological constancy at the load. There were no significant changes in cyclists', heart rate during the 30-minute cycling exercise at constant intensity. The subjects' average heart rate was between 139-170 bpm. They began the cycling test with the heart rate of 90 bpm and finished the test with the heart rate at $162 \mathrm{bpm}$. Average heart rate of all subjects during the test was $153 \pm 2.7 \mathrm{bpm}$. 
Over the past years, several studies have measured knee extensor muscle strength loss after prolonged exercise. The results of the present study also showed the significant reduction in isometric MVC force of the knee extensor muscles after the cycling exercise. MVC force of knee extensor muscles before the test was $647.7 \pm 27.7 \mathrm{~N}$. After cycling exercise, MVC force decreased significantly $(9.9 \%$, $\mathrm{p}<0.001$ ) compared to the initial level. MVC loss is consistent with the previous studies conducted with similar cycling intensity and duration [1, 14]. This decrease in maximal isometric voluntary contraction force after exercise may involve processes associated with the central command of contraction [3], as well as peripheral processes of intramuscular electrical and mechanical failure [5]. The failure to maintain the required force can also be related to neural factors (muscle activation) associated with excitation, recruitment and firing rate of motor units. It was noted that corticomotor excitability increases during a sustained submaximal voluntary contraction [11]. This decrease in MVC force generation capacity after fatiguing exercise may similarly involve processes associated with the central command of contraction $[2,13]$, as well as peripheral processes of intramuscular electrical and mechanical failure [5].

We concluded that based on electromyography power spectrum median frequency, 30-minute cycling exercise at constant intensity caused a marked fatigue of erector spinae muscle with no significant fatigue-induced changes in knee extensor and flexor muscles. Subjectively assessed fatigue was increased at the end of exercise and there was noted a marked decrease in voluntary force generation capacity of the knee extensor muscles after the end of exercise.

\section{REFERENCES}

1. Balasubramanian V., Jayaraman S. (2009) Surface EMG based muscle activity analysis for aerobic cyclist. Bodywork Mov. Ther. 13: $34-42$

2. Bigland-Ritchie B. (1981) EMG and fatigue of human voluntary and stimulated contractions. Ciba Found. Symp. 82: 130-156

3. Bigland-Ritchie B., Furbush F., Woods J. J. (1986) Fatigue of intermittent submaximal voluntary contractions: central and peripheral factor in different muscles. J. Appl. Physiol. 61: 421-429

4. Borg G. A. V. (1998) Borg's Perceived Exertion and Pain Scales. Champaign IL: Human Kinetics 
5. Brody L. R., Pollock M. T., Roy S. H., De Luca C. J., Celli B. (1991) $\mathrm{pH}$-induced effects on median frequency and conduction velocity of myoelectric signal. J. Appl. Physiol. 71: 1878-1885

6. Buttelli O., Seck D., Vandewalle H., Jouanin J. C., Monod H. (1996) Effect of fatigue on maximal velocity and maximal torque during short exhausting cycling. Eur. J. Appl. Physiol. 73: 175-179

7. Clark B. C., Collier S. R., Manini T. M., Ploutz-Snyder L. L. (2005) Sex differences in muscle fatigability and activation patterns of the human quadriceps femoris. Eur. J. Appl. Physiol. 94: 196-206

8. Creer A. R., Ricard M. D., Conlee R. K., Hoyt G. L., Parcell A. C. (2004) Neural, metabolic, and performance adaptations to four weeks of high intensity sprint-interval training in trained cyclists. Int. J. Sports Med. 25: 92-98

9. Edwards, R. H. T, Hill D. K, Jones D. A., Merton P. A. (1977) Fatigue of long duration in human skeletel muscle after exercise. J. Physiol. Lon. 272: 769-778

10. Edwards, R. H. T. (1981) Human muscle function and fatigue. In: Porter R. and Whelan J. (eds.). Human Muscle Fatigue: Physiological Mechanisms. London: Pitman Medical. 1-18

11. Gandevia S. C. (2001) Spinal an supraspinal factors in human muscle fatigue. Physiol. Rev. 81: 1725-1789

12. Masuda K., Masuda T., Sadoyama T., Inaki M., Katsuta S. (1999) Changes in surface EMG parameters during static and dynamic fatiguing contractions. J. Electromyogr. Kinesiol. 9: 39-46

13. Mckay W. B., Tuel S. M, Sherwood A. M. Stokic D. S. Dimitrijevic M. R. (1995) Focal depression of cortical excitability induced by fatiguing muscle contraction: a transcranial magnetic stimulation study. Exp. Brain Res. 105: 276-282

14. Millet G. Y., Lepers R. (2004) Alterations of neuromuscular function after prolonged running, cycling and skiing exercises. Sports Med. 34: $105-116$

15. Pérez-Landaluce J., Fernández-García B., Rodríguez-Alonso M., García-Herrero F., García-Zapico P., Patterson A. M., Terrados N. (2002) Physiological differences and rating of perceived exertion (RPE) in professional, amateur and young cyclists. J. Sports Med. Phys. Fitness 42: 389-395

16. Pääsuke M., Ereline J., Gapeyeva H. (1999) Neuromuscular fatigue during repeated exhaustive submaximal static contractions of knee extensor muscles in endurance-trained, power-trained and untrained men. Acta Physiol. Scand. 166: 319-326

17. Rasmussen P., Secher N. H., Petersen N. T. (2007) Understanding central fatigue: where to go? Exp. Physiol. 92: 369-370 
18. Sesboüé B., Guincestre J. Y. (2006) Muscular fatigue. Ann. Readapt. Med. Phys. 49: 348-354

19. Stulen F., Deluca C.J. (1981) Frequency parameters of the myoelectric signal as a measure of muscle conduction velocity. IEEE Trans. Biomed. Eng. 28: 515-523

\section{Correspondence to:}

Laura Lepasalu

University of Tartu, Institute of Exercise Biology and Physiotherapy, 4 Ujula Street, 51008 Tartu

Estonia

E-mail: lepasalu@hotmail.com 